\title{
Les prothèses gonflables dans le traitement de l'impuissance : résultats de 80 implantations
}

\author{
D. Rossi, F. Bladou, D. Ayuso, C. Rattier, M. Hermanowicz, G. Serment
}

Service d'urologie, Hôpital Salvator, 249 Bd. de Sainte Marguerite, 13009 Marseille

\section{RESUME}

La chirurgie prothétique de l'impuissance a été transformée par l'apparition des prothèses gonflables détrônant les modèles semi-rigides plus anciens. L'engouement suscité par cette nouvelle technique a aujourd'hui laissé place à des interrogations sur les résultats fonctionnels et les compli. cations de cette chirurgie rarement étudiés de manière concomitante dans la littérature. Nous rapportons notre expérience à propos de 80 mises en place de prothèses gonflables posées entre octobre 1987 et octobre 1994. Le suivi moyen est de 3 ans, l'évaluation des résultats objectifs (fonctionnement mécanique de la prothèse, complications) et subjectifs (sexualité des patients), porte sur 68 patients. Les résultats sont :

- $54,5 \%$ d'anomalies de fonctionnement,

- 7\% d'infections de la prothèse,

- 27,5\% d'ablations du matériel.

La majorité des patients se déclarent satisfaits bien que seulement $65 \%$ ont repris une activité sexuelle régulière.

Mots clés : impuissance; prothèse pénienne.

L'avènement des injections intracaverneuses de drogues vasoactives $[8,19,31$, 34] a diminué la part de la chirurgie dans le traitement de l'impuissance organique. Parmi les traitements chirurgicaux la mise en place d'une prothèse pénienne est une intervention de dernier recours $[1,7,8,34]$. Depuis l'apparition en 1973 des prothèses de verge gonflables $[3,29]$ détrônant les modèles semi-rigides plus anciens, de nombreux articles ont été publiés étudiant les complications mécaniques et infectieuses ou la sexualité des patients. Nous rapportons notre expérience dans ces différents domaines à propos de 80 mises en place de prothèses péniennes gonflables.

\section{MATERIEL ET METHODES}

Nous avons implanté d'octobre 1987 à octobre 1994, 80 prothèses péniennes gonflables.

Le matériel utilisé a été :

- 40 prothèses MENTOR Mark 1 et 2

- 27 prothèses MENTOR Alpha 1.

- 13 prothèses AMS Hydroflex.

L'âge moyen des patients est de 58 ans (25 à 78 ans). Les pathologies responsables de l'impuissance étaient variées avec parmi celles-ci peu de causes psychogènes pures (Tableau 1).

Le bilan initial a comporté :

- un interrogatoire et un examen clinique traditionnels,

- un bilan biologique (glycémie, testostéronémie, prolactinémie, dosage du PSA), 
- un Doppler pulsé des vaisseaux péniens et des membres inférieurs avec test pharmacologique sensibilisateur (papavérine 20 milligrammes puis alprostadil 10 microgrammes depuis 2 ans)

- un entretien sexologique.

- des tests intracaverneux (TIC) à doses croissantes (Papavérine puis alprostadil).

En deuxième intention une cavernographie couplée à une cavernométrie était demandée lorsqu'il existait une anomalie évocatrice d'une pathologie veino - caverneuse (érection instable typique, doppler artériel et bilan hormonal normaux ).

Les critères pour la mise en place d'une prothèse pénienne ont été les suivants :

- Tests pharmacologiques intracaverneux négatifs à 4 reprises et à fortes doses (Papavérine 80 milligrammes, Alprostadil 40 microgrammes).

- Refus ou échec des auto injections quand celles-ci étaient la seule ressource thérapeutique.

L'indication étant posée, plusieurs entretiens avec présentation et manipulation du matériel en présence de la partenaire ont été réalisés afin de s'assurer de la motivation du couple et de sa capacité à utiliser un modèle gonflable.

L'implantation chirurgicale de la prothèse a été effectuée sous antibiothérapie prophylactique péri-opératoire, par un abord pénoscrotal ou sous pubien associé à une incision abdominale pour mise en place du réservoir.

Les patients ont été revus en consultation à 1 mois, 3 mois, 6 mois, 1 an, puis tous les ans. Lors de ces consultations le médecin a apprécié la facilité de gonflage et de dégonflage ainsi que la qualité de la rigidité. La sexualité du patient a été évaluée par l'interrogatoire du patient en présence de la partenaire.
Tableau 1 : Indications de mise en place d'une prothèse de verge gonflable.

Artérite

21 cas

Diabète

14 cas

Fuite veineuse

7 cas

Atteinte neurologique

3 cas

Maladie de LAPEYRONIE

4 cas

Fibrose caverneuse après priapisme 8 cas

Traitement hormonal

6 cas

Chirurgie pelvienne élargie

Traumatisme périnéal grave

Psychogène

13 cas

2 cas

2 cas

\section{RESULTATS}

Le suivi moyen des patients est de 3 ans (6 mois à 7 ans). Les suites opératoires immédiates ont été simples dans la majorité des cas (57 cas sur 80 ). Nous avons observé 20 complications mineures spontanément résolutives (hématome, œdème, rétention d'urine) ; 3 patients ont présenté une infection sévère d'emblée nécessitant l'explantation rapide.

L'évaluation des résultats à long terme porte sur 68 patients (3 explantations précoces et 9 patients perdus de vue).

Les résultats sur le fonctionnement mécanique des prothèses sont exposés dans le tableau 2. Sur les 37 anomalies de fonctionnement retrouvées, $12(17,5 \%)$ ont imposé une réintervention : ablation d'emblée de la prothèse dans 3 cas et révision dans 9 cas. Parmi ces 9 prothèses révisées, 5 ont dû être explantées secondairement avec un délai moyen de 6 mois.

Les autres causes d'ablation de prothèses ont été : 6 ulcérations cutanées et 5 syndromes douloureux péniens évoluant plusieurs mois après la mise en place de la prothèse. Il s'agissait d'infections chroniques (découverte en per opératoire d'une collection péri prothétique).

Nous n'avons pas retrouvé d'étiologie de l'impuissance favorisant la survenue d'une anomalie de fonctionnement où l'ablation de la prothèse. 
Tableau 2 : Résultats mécaniques évalués au 6 ème mois postopératoire, chez 68 patients (3 infections précoces; 9 patients perdus de vue).

- 31 prothèses fonctionnants parfaitement :

$45,5 \%$

- 37 anomalies de fonctionnement

- 16 : rigidité insuffisante

$23,5 \%$

- 21 : dysfonctionnement

$31 \%$

* 3 prothèses bloquées en érection

$54,5 \%$

* 15 anomalies au gonflage

* 3 scléroses autour de la pompe

$31 \%$

$6 \%$

Tableau 3 : Causes des révisions chirurgicales et des ablations de matériel après mise en place d'une prothèse de verge gonflable.

80 PROTHESES GONFLABLES

09 patients perdus de vue

71 patients avec un suivi

moyen de 3 ans

\section{Ablation \\ De 1ère intention \\ Révision chirurgicale}

Ablation

secondaire
- 3 Infections précoces :

3

- 12 réinterventions pour anomalie de fonctionnement :

- 5 anomalies de gonflage

- 4 lâchages de raccords

- 3 scléroses autour de la pompe

- 5 syndromes doul. (inf. chron.) :

- 6 érosions cutanées :
2

0

1

5

6
0

32

42

21

0
0

2

2

TOTAL

17

9

5

Au total 22 prothèses sur 80 ont été explantées soit $27,5 \%$ (Tableau 3 ). Le délai moyen d'ablation de la prothèse est de 13 mois (15 jours à 2 ans).

Les résultats subjectifs à savoir l'analyse de la sexualité des patients porteurs d'une prothèse depuis au moins 1 an sont exposés dans le tableau 4.

\section{DISCUSSION}

Le développement des prothèses péniennes gonflables introduites par Scott [29] a ouvert une nouvelle ère dans le traitement de l'impuissance. Cependant l'enthousiasme initial a été tempéré par un nombre important de complications essentiellement d'origine mécanique et infectieuse [9, 12 , $15,18,20,22,24,29,36]$.
Tableau $4:$ Résultats fonctionnels : Analyse de la sexualité des patients au 6 ème mois postopératoire. Les résultats sont évalués sur l'interrogatoire en présence de la partenaire.

\begin{tabular}{lr} 
Patients satisfaits & $70 \%$ \\
Reprise d'une activité sexuelle & $65 \%$ \\
Diminution du plaisir & $60 \%$ \\
Diminution de la longueur du pénis & $75 \%$ \\
Douleurs & $6 \%$ \\
& \\
Libido diminuée & $50 \%$ \\
Orgasme altéré & $60 \%$ \\
\hline
\end{tabular}


Seulement $45,5 \%$ de nos patients ont un résultat mécanique parfait (Tableau 2 ). Ce chiffre est inférieur aux données de la littérature $[4,9,15,18,20,25,30,35,36]$. Cependant il est important de noter que contrairement à de nombreux auteurs qui entendent par dysfonctionnement une altération de la fonction imposant une révision chirurgicale, dans notre série les anomalies de fonctionnement minimes comme un dégonflage imparfait, ont été comptabilisées comme dysfonctionnement. Notre taux de réintervention pour problème mécanique grave est de $17,5 \%$ chiffre comparable aux données de la littérature.

Les ruptures de cylindres ou de réservoir, les dysfonctionnements de la pompe et les fuites au niveau des raccords sont les causes de réinterventions les plus fréquentes [8, 18, 20, 24, 29]. Trois de nos patients ont développé une sclérose autour de la pompe scrotale empêchant son activation. Ceci est au volume important de la pompe dans le modèle de première génération de Mentor.

Concernant les complications infectieuses nous observons également des taux un peu plus élevés que ceux de la littérature $[2,4$,
$6,12,14,16,18,22,23,26,31-33]$ (Tableau $6)$. Il faut remarquer que si les 3 infections précoces ont été de diagnostic facile, le diagnostic des 5 autres infections a été fait lors de la réintervention pour un syndrome douloureux pénien chronique évoluant depuis plusieurs mois.

Devant cette constatation, nous pensons que des douleurs péniennes chroniques persistant plusieurs mois après l'intervention doivent faire évoquer une infection sous jacente.

Il est plus difficile de comparer nos résultats subjectifs liés à la sexualité du patient aux données de la littérature. Dans notre série $70 \%$ des patients des patients interrogés par le chirurgien sont satisfaits et seulement $65 \%$ d'entre eux ont repris une activité sexuelle régulière. Ces chiffres sont inférieurs à ceux publiés dans la littérature $[10,11,17,21,25,28]$. Cette différence s'explique par le fait que beaucoup d'études reposent sur l'analyse de questionnaires expédiés aux opérés et que seulement 50 à $70 \%$ des patients répondent à ces questionnaires.

Le choix entre un modèle gonflable et un modèle semi-rigide doit donc être prudem-

Tableau 5 : Dysfonctionnement et réinterventions après mise en place d'une prothèse de verge gonflable : Données de la littérature.

\begin{tabular}{lccc}
\hline Auteurs & $\begin{array}{c}\text { Nombre } \\
\text { de patients }\end{array}$ & $\begin{array}{c}\text { Reinterventions } \\
\text { pour dysfonctionnement }\end{array}$ & $\begin{array}{c}\text { Reinterventions } \\
\text { Total }\end{array}$ \\
\hline Kaufman 1982 & 84 & $44 \%$ & $54 \%$ \\
Joseph 1984 & 88 & $46,6 \%$ & $?$ \\
Gregory 1984 & 184 & $43 \%$ & $?$ \\
Engel 1986 & 116 & $9,5 \%$ & $?$ \\
Furlow 1988 & 120 & $10 \%$ & $?$ \\
Brooks 1988 & 137 & $7,3 \%$ & $12,4 \%$ \\
Pedersen 1988 & 52 & $19,2 \%$ & $27 \%$ \\
Wilson 1988 & 395 & $19,2 \%$ & $31,8 \%$ \\
Woodworth 1991 & 266 & $22,1 \%$ & $?$ \\
Steinkolhl 1991 & 46 & $?$ & $21,7 \%$ \\
Goldstein 1993 & 112 & $4 \%$ & $9 \%$ \\
Fein 1994 & 138 & $3,5 \%$ & $5 \%$ \\
Notre Série 1995 & 80 & $17,5 \%$ & $32 \%$ \\
\hline
\end{tabular}


Tableau 6 : Complications infectieuses après mise en place d'une prothèse de verge gonflable : Données de la littérature.

\begin{tabular}{lccr}
\hline Auteur & Année & $\begin{array}{c}\text { Nb. de } \\
\text { prothèses }\end{array}$ & $\begin{array}{r}\% \\
\text { d'infec. }\end{array}$ \\
\hline Furiow & 1979 & 175 & $3,4 \%$ \\
Kaufman & 1982 & 84 & $3,4 \%$ \\
Thomala & 1987 & 150 & $8 \%$ \\
Montague & 1987 & 246 & $3,7 \%$ \\
Brooks & 1988 & 137 & $2,1 \%$ \\
Merill & 1989 & 150 & $\mathbf{1 , 3 \%}$ \\
Radomsky & 1992 & 107 & $1,8 \%$ \\
Bischop & 1992 & 90 & $5,5 \%$ \\
Goldstein & 1993 & 112 & $2 \%$ \\
Fein & 1994 & 138 & $1,5 \%$ \\
Notre Série & 1995 & 80 & $7 \%$ \\
\hline
\end{tabular}

ment mesuré du fait de la fréquence des complications mécaniques sur les modèles sophistiqués. Ceci d'autant plus qu'une sélection rigoureuse des patients ne permet pas toujours de préjuger de leur sexualité future.

La prothèse de verge gonflable reste cependant le modèle de référence. Sa fiabilité s'améliore du fait de la fabrication de prothèses monocorps $[11,13,14,27]$. Reste le problème du coût $d u$ matériel qui doit être comparé au traitement par injections intracaverneuses [5].

\section{REFERENCES}

1. BONDIL P., RIGOT J.M. : Les indications de prothèses péniennes. Contracept. Fertil. Sexual., 1986, 14, 1025-1029.

2. BISHOP J.R., MOUL J.W., SIHELNIC S.A., PEPPAS D.S., GORMLEY T.S. and McLEOD D.G. : Use of glycosylated hemoglobin to identify diabetics at high risk for penile periprosthetic infections. J. Urol., 1992, 147, 386-388.

3. BRETAN P.N., M.D.,Jr. : History of the prosthetic treatment of impotence. Urol. Clin. N. Amer. 1989, $16,1-5$.

4. BROOKS M.B. : 42 months of experience with MENTOR inflatable penile prothesis. J. Urol.;1988, 139, 48-49.
5. BUCH JP, ZORN BH, TAYLOR RJ. : Cost - benefit analysis of pharmacologic erection program versus penile prosthesis. Urology, 1991, 37(2), 116-118.

6. CARSON C.C., and ROBERTSON C.N. : Late hematogenous infection of penile prostheses. J. Urol., 1988, 139, 50-52.

7. CUMMING J. and PRYOR J.P. : Treatment of organic impotence. British Journal of Urology., $1991,67,640-643$

8. DELAGER S. THOMAS M. : L'impuissance: possibilités thérapeutiques. Ann. Med. Interne., 1990, 4,367-373.

9. ENGEL R.M.E, SMOLEV J.K. and HACKLER R. : Experience with the MENTOR inflatable penile prothesis. J. Urol., 1986, 135, 1181-1182.

10. FALLON B. GHANEM H. : Sexual performance and satisfaction with penile prosthesis in impotence of various etiologies. Int. J. Impotence Res., $1990,2,35-42$.

11. FEIN RL. : GFS Mark II inflatable penile prosthesis : four - year clinical study. Urology, 1994, 43(2), 209-213.

12. FURLOW W.L., GOLDWASSER B. and GUNDIAN J.C. : Implantation of AMS 700 penile prosthesis: long term results. J. Urol., 1988, 139, 741742.

13. GARBER BB. : Mentor alpha 1 inflatable penile prosthesis : patient satisfaction and device reliability. Urology, 1994, 43(2), 214-217.

14. GOLDSTEIN I, BERTERO EB, KAUFMAN JM, WITTEN FR et al : Early experience with the first pre-connected 3-piece inflatable penile prosthesis : the Mentor alpha 1. J. urol, 1993, 150(6), 18141818.

15. GREGORY J.G., PURCELL M.H. and STANDEVEN J. : The inflatable penile prosthesis: failure of the rear tip extender in reducing the incidence of cylinder leakage. J. Urol., 1984, 131, 668-669.

16. KABALIN J.N. and KESSLER R. : Infectious complications of penile prosthesis surgery. J. Urol. 1988, 139, 953-955.

17. KAUFMAN J.J., BOXER R.J., BOXER B. and QUINN M.C. : Physical and psychological results of penile prothesis: A statical survey. J. Urol., $1981,126,173-175$.

18. KAUFMAN J.J., LINDNER A. and RAZ S. : Complication of penile prothesis surgery for impotence. J. Urol., 1982, 128, 1192-1194.

19. LUE F.T., TANAGHO E.A. : Physiology of erection and pharmacological management of impotence. Urology, 1987, 137, 829-835. 
20. JOSEPH D.B., BRUSKEWITZ R.C. and BENSON R.C. : Long term evaluation of the inflatable penile prothesis. J. Urol., 1984, 131, 670-673.

21. McLAREN R.H. and BARRETT D. M. : Patient and partner satisfaction with the AMS 700 penile prosthesis. J. Urol., 1992, 147, 63-65.

22. MERRILL D.C.: MENTOR inflatable prothesis. Urol. Clin. N. Amer. 1989, 16, 7-12.

23. MONTAGUE D.K. : Periprosthetic infections. J. Urol., 1987, 138, 68-69.

24. MONTAGUE D.K. : Penile prothesis, an overview. Urol. Clin. N. Amer., 1989, 16, 7-12.

25. PEDERSEN B., TIEFER L., RUIZ M. and MELMAN A. : Evaluation of patients and partners 1 to 4 years after penile prosthesis surgery. J. Urol., $1988,139,956-958$.

26. RADOMSKI S.B., and HERSCHORN S. : Risk factors associated with penile prosthesis infection. J. Urol., 1992, 147, 383-385.

27. RANDRUP E, WILSON S, MOBLEY D, SUAREZ G, MEKRAS G, BAUM N. : Clinical experience with Mentor alpha 1 inflatable penile prosthesis. Report of 333 cases. Urology, 1993, 42, 305-310.

28. SCHLAMOWITZ K.E., BEUTLER R.E., SCOTT F.B., KARAKAN I. and WARE S. : Reaction of the implantation of inflatable prothesis among psychogenally and organically impotent men. J. Urol., 1983, 129, 295-298.

29. SCOTT F.B., BRADLEY W.E. and TIMM G.W. : Management of erectile impotence. Use of implantable prosthesis. Urology, 1973, 1, 80.

30. STEINKOHL W.B. and LEACH G.E. : Mechanical complications associated with MENTOR inflatable penile prosthesis. Urology, 1991, 38, 32-34.

31. THOMALA J.V., THOMPSON S.T., ROWLAND R.G. and MULCAHY J.J. : Infectious complications of penile prosthetic implants. J. Urol., 1987, 138, 65-67.

32. VIRAG R. : Intracavernous injection of papaverine for erectile failure. Lancet, 1982, 2, 938.

33. WALTERS F.P., NEAL D.E., REGE A.B., GEORGE W.J., RICCI M.J. and HELLSTROM W.J. N. : Cavernous tissue antibiotic levels in penile prosthesis surgery. J. Urol., 1992, 147, 1282-1284.

34. WESPES E. : Impuissance organique masculine. Acta. Urol. Belg. 1987, 55, 1-330.

35. WILSON K.S., WAHMAN G.E. and LANGE J.L. : Eleven years of experience with the inflatable penile prosthesis. J. Urol., 1988, 139, 951-952.

36. WOODWORTH B.E., CARSON C.C. and WEBSTER G. D. : Inflatable penile prosthesis: effect of device modification on functionnal longevity. Urology., 1991, 38, 533-536.
ABSTRACT
D. Rossi, F. Bladou, D. Ayuso, C. Rat- TIER, M. Hermanowicz, G. Serment

The prosthetic surgery of impotence has been transformed by the onset of inflatable prostheses which have superseded the old semi-rigid designs.

\section{Objective}

The goal of this study is the evaluation of the functionnal results and the complications of this type of surgery. Such questions have been poorly discussed in the literature.

\section{Methods}

We report our experience on 80 inflatable prostheses that were implanted between october 1987 and october 1994. The mean follow-up is 3 years and the assessment of the objective (mechanical functioning of the prosthesis and complications) and sujective results (sexuality of the patients) is carried out on 68 patients.

\section{Results}

The results are the following :

- $54.5 \%$ of functioning disturbances,

- 7\% of infections,

- $27.5 \%$ of prosthesis removals.

Most of the patients considered they were satisfied although only $65 \%$ returned to a regular sexual activity.

\section{CONCLUSIONS}

The inflatable penile prostheses remain nevertheless the designs of reference. Their reliability is increasing because of the manufacture of monobloc designs. The cost of such a device remains, and deserves to be compared with that of the intra-cavernous injections.

Key words : impotence; penile prosthesis. 